\title{
Critical Currents, Magnetization and Microwave Absorption of $\left(\mathrm{Tl}_{0.5} \mathrm{~Pb}_{0.5}\right) \mathrm{Sr}_{2}\left(\mathrm{Ca}_{0.8} \mathrm{Gd}_{0.2}\right) \mathrm{Cu}_{2} \mathrm{O}_{z}$ Superconductor
}

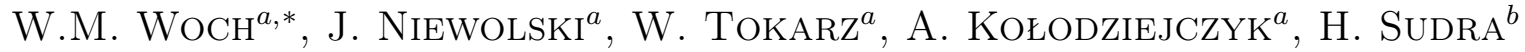 \\ AND G. GRITZNER ${ }^{b}$ \\ ${ }^{a}$ Solid State Physics Department, Faculty of Physics and Applied Computer Science \\ AGH University of Science and Technology, al. Mickiewicza 30, 30-059 Kraków, Poland \\ ${ }^{b}$ Institute for Chemical Technology of Inorganic Materials, Johannes Kepler University \\ Altenbergerstr. 69, 4040 Linz, Austria
}

\begin{abstract}
The bulk $\left(\mathrm{Tl}_{0.5} \mathrm{~Pb}_{0.5}\right) \mathrm{Sr}_{2}\left(\mathrm{Ca}_{1-x} \mathrm{Gd}_{x}\right) \mathrm{Cu}_{2} \mathrm{O}_{z}$ superconductors with $x=0.1,0.2$ and 0.3 have unique and very interesting magnetic and electrical properties. In this paper we focused our attention on the superconductor with gadolinium content $x=0.2$. We measured the magnetic field dependences of the transport critical currents, the microwave absorption as well as the magnetization for different temperatures. The critical temperature of this sample is $T_{\mathrm{c}}=105.3 \pm 0.2 \mathrm{~K}$ and the transition width $\Delta T_{90 \%-10 \%}=3.6 \pm 0.2 \mathrm{~K}$. The critical current of this superconductor is $J_{\mathrm{c}}=1820 \pm 10 \mathrm{~A} / \mathrm{cm}^{2}$ at $77 \mathrm{~K}$. The data were analyzed within the frame of the Bean and Kim models as well as the percolation model.
\end{abstract}

PACS numbers: 74.81.Bd, 74.25.Sv, 74.81.Fa

\section{Introduction}

Thallium superconductors belong to the cuprate based superconductors which possess alterable superconducting properties. Their critical temperatures, critical currents and irreversibility fields vary in wide ranges for different elemental substitutions and may be adjusted to specific needs [1-4].

In this paper the properties of the bulk $\left(\mathrm{Tl}_{0.5} \mathrm{~Pb}_{0.5}\right) \mathrm{Sr}_{2}\left(\mathrm{Ca}_{1-x} \mathrm{Gd}_{x}\right) \mathrm{Cu}_{2} \mathrm{O}_{z} \quad$ superconductor are presented. The sample with $x=0.2$ was chosen to examine its unique inter-granular properties by means of the critical currents, the magnetisation and the microwave absorption measurements.

The transport critical current flows through the grains as well as through the inter-grain links which may be treated as Josephson junctions (weak links). The critical current is limited by the weakest junctions on the percolate path of the current.

The magnetic field dependence of the critical current was firstly introduced by Kim et al. [5] for hard superconductors. They assumed that the reverse of the critical current is proportional to the power series expansion

$$
\frac{a}{J}=H_{0}+H+a_{1} H^{2}+a_{2} H^{3}+\ldots,
$$

where $a$ and $H_{0}$ are material constants which can be determined from experiment and $H$ is the applied field. If the coefficients $a_{1}, a_{2}, \ldots$ are sufficiently small, Eq. (1) reduces to

\footnotetext{
* corresponding author; e-mail: wmwoch@agh.edu.pl
}

$$
\frac{a}{J}=H_{0}+H \text {. }
$$

This is the central result of the Kim critical state model that describes the critical current versus the applied magnetic field of the hard superconductors.

Unfortunately, Eq. (2) does not fit the magnetic dependences of the critical current of the high temperature superconductors (HTS). Therefore, the extended models should be developed. One of them includes the higher power quadratic term of the expansion (1) and can be written as follows [6, 7]:

$$
J_{\mathrm{c}}=J_{\mathrm{c} 0}\left[1+\left(\frac{H}{H_{K}}\right)\right]^{-n},
$$

where $J_{\mathrm{c} 0}$ is the critical current at the zero magnetic field, the parameter $H_{K}$ is material constant and exponent $n$ is the fitting parameter. This model was called as a generalized critical state model in the paper [6] and usually well describes thin films and single crystals where the weak links can be neglected [1, 7].

The magnetic field dependence of the critical current can be also expressed by the percolation critical state model. This model assumes a weak link network of the Josephson coupled grains with a critical current that has two components: the field-independent component and a field dependent component that decays exponentially with the applied magnetic field [8]:

$$
J_{\mathrm{c}}=J_{c 0}^{*}\left[\exp \left(-\frac{H}{H_{e}}\right)+\beta\right],
$$

where $J_{c 0}^{*}(1+\beta)$ is the critical current at zero magnetic field, $H_{e}$ is a phenomenological parameter connected with weak link network properties and $\beta$ is the fitting parame- 
ter. According to the generalized critical state model [6], Eq. (4) can be calculated from Eq. (3) if $H / H_{K} \ll 1$ and $n \gg 1$ for negligible $\beta$. Equation (4) fits better critical currents of bulk superconductors.

\section{Experiment}

The samples were prepared by a wet chemical gel technique followed by a special heat-treatment procedure [9]. Some more magnetic and electrical properties of the bulk $\left(\mathrm{Tl}_{0.5} \mathrm{~Pb}_{0.5}\right) \mathrm{Sr}_{2}\left(\mathrm{Ca}_{1-x} \mathrm{Gd}_{x}\right) \mathrm{Cu}_{2} \mathrm{O}_{z}$ superconductors with $x=0.1,0.2$ and 0.3 were published in our previous papers [4].

The transport critical currents measurements were carried out at $77 \mathrm{~K}$ using the dc four-point technique. The electrical contacts were made by silver paint, which were sintered at $300{ }^{\circ} \mathrm{C}$. The measurements of the voltage drop versus flowing current for different values of the applied dc magnetic fields were carried out using the four-point ac method of $7 \mathrm{~Hz}$ current frequency. The transport critical currents were obtained employing the $1 \mu \mathrm{V} \mathrm{cm}-1$ criterion.

The real and imaginary ac susceptibility as a function of ac magnetic fields was measured by a standard mutual inductance bridge operating at the frequency of $189 \mathrm{~Hz}$. A Stanford SR 830 lock-in nanovoltmeter served both as a source for the ac current for the coil which produced the ac magnetic field and as a voltmeter of the bridge. The applied dc magnetic field was produced by a copper solenoid immersed in a liquid nitrogen bath. The temperature from $77 \mathrm{~K}$ to $300 \mathrm{~K}$ was monitored by a Lake Shore temperature controller employing a chromel-gold$0.07 \% \mathrm{Fe}$ thermocouple with an accuracy of $\pm 0.05 \mathrm{~K}$ for both experimental setups.

The magnetization measurements were carried out on a Lake Shore Vibrating Sample Magnetometer combined with a Janis cryostat.

The magnetically modulated microwave absorption (MMMA) measurements were carried out using a home-made microwave spectrometer operating at $9.5 \mathrm{GHz}[10]$. The sample was placed at the centre of cavity with $\mathrm{TE}_{102}$ mode in maximum of microwave magnetic field. The incident microwave power was $2 \mathrm{~mW}$.

For magnetization as well as for MMMA measurements the applied dc magnetic field was produced by electromagnet with a pair of correcting Helmholtz coils that cancelled the residual remanence of electromagnet.

\section{Results and discussion}

X-ray diffraction analysis of bulk $\left(\mathrm{Tl}_{0.5} \mathrm{~Pb}_{0.5}\right) \mathrm{Sr}_{2}\left(\mathrm{Ca}_{1-x} \mathrm{Gd}_{x}\right) \mathrm{Cu}_{2} \mathrm{O}_{z} \quad$ superconductors yielded practically phase pure samples with tetragonal structure (space group $P / 4 \mathrm{mmm}$ ). The microstructure of the bulk material shows round shaped crystallites, partly melted at the edges, with diameters around $1 \mu \mathrm{m}$ that were typical for the Tl-1212 phase [4].

For the investigation the sample with $x=0.2$ was selected because it has highest critical temperature as well as the critical current density at $77 \mathrm{~K}$. The characteristic critical temperatures and the transition width were obtained from the magnetoresistance measurements at zero applied magnetic field. The critical current densities were obtained from the susceptibility measurements using the Bean model and from the transport measurements at $77 \mathrm{~K}$ by the method described in $[1,4]$.

The stoichiometric coefficients, the lattice parameters, the transition temperatures, the transition width and the critical current density calculated from the Bean model as well as the critical current densities obtained from transport measurements are collected in Table.

TABLE

The stoichiometric coefficients, lattice parameters, transition temperatures, transition width, and critical current density calculated from the Bean model as well as critical current densities obtained from transport measurements of $\left(\mathrm{Tl}_{0.5} \mathrm{~Pb}_{0.5}\right) \mathrm{Sr}_{2}\left(\mathrm{Ca}_{0.8} \mathrm{Gd}_{0.2}\right) \mathrm{Cu}_{2} \mathrm{O}_{z}$ bulk superconductors.

\begin{tabular}{c|c|c|c|c|c|c|c|c|c}
\hline \hline$x$ & $z$ & $\begin{array}{c}a \\
{[\AA]}\end{array}$ & $\begin{array}{c}c \\
{[\AA]}\end{array}$ & $\begin{array}{c}T_{\mathrm{c} 0} \\
{[\mathrm{~K}]}\end{array}$ & $\begin{array}{c}T_{\text {c50\% }} \\
{[\mathrm{K}]}\end{array}$ & $\begin{array}{c}T_{\text {c onset }} \\
{[\mathrm{K}]}\end{array}$ & $\begin{array}{c}\Delta T_{90 \%-10 \%} \\
{[\mathrm{~K}]}\end{array}$ & $\begin{array}{c}J_{\mathrm{c}}^{\text {Bean model }} \\
{\left[\mathrm{A} / \mathrm{cm}^{2}\right]}\end{array}$ & $\begin{array}{c}J_{\mathrm{c}}^{\text {transport }} \\
{\left[\mathrm{A} / \mathrm{cm}^{2}\right]}\end{array}$ \\
\hline 0.2 & 7.03 & 3.8038 & 12.1071 & 100.1 & 105.3 & 109.7 & 3.60 & 1738 & 1820
\end{tabular}

The absorption of ac susceptibility showed only one maximum. It means that the critical current of the grains dominates in these superconductors. On the other hand, the transport critical current depends mainly on the properties of inter-granular junctions. These values are very similar, so one can suggest that the inter-granular links are strong and their properties are similar to the grains ones.
In order to check the properties of both the grains and inter-grain links the critical current, magnetization as well as the microwave absorption of $\left(\mathrm{Tl}_{0.5} \mathrm{~Pb}_{0.5}\right) \mathrm{Sr}_{2}\left(\mathrm{Ca}_{0.8} \mathrm{Gd}_{0.2}\right) \mathrm{Cu}_{2} \mathrm{O}_{z}$ superconductor were measured.

The critical current as a function of the applied magnetic field is shown in Fig. 1. The experimental points (open circles) are fitted using the Kim Eq. (3) as well 


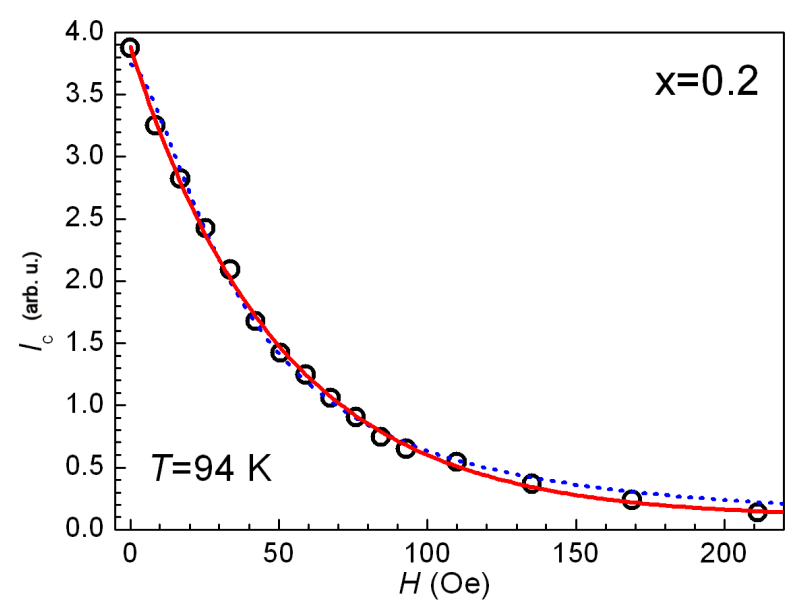

Fig. 1. Critical current

of $\left(\mathrm{Tl}_{0.5} \mathrm{~Pb}_{0.5}\right) \mathrm{Sr}_{2}\left(\mathrm{Ca}_{0.8} \mathrm{Gd}_{0.2}\right) \mathrm{Cu}_{2} \mathrm{O}_{z} \quad$ superconductor versus the applied magnetic field at $94 \mathrm{~K}$ (open circles). Dotted line is the fitting of Eq. (3) and solid line is the fitting of Eq. (4).

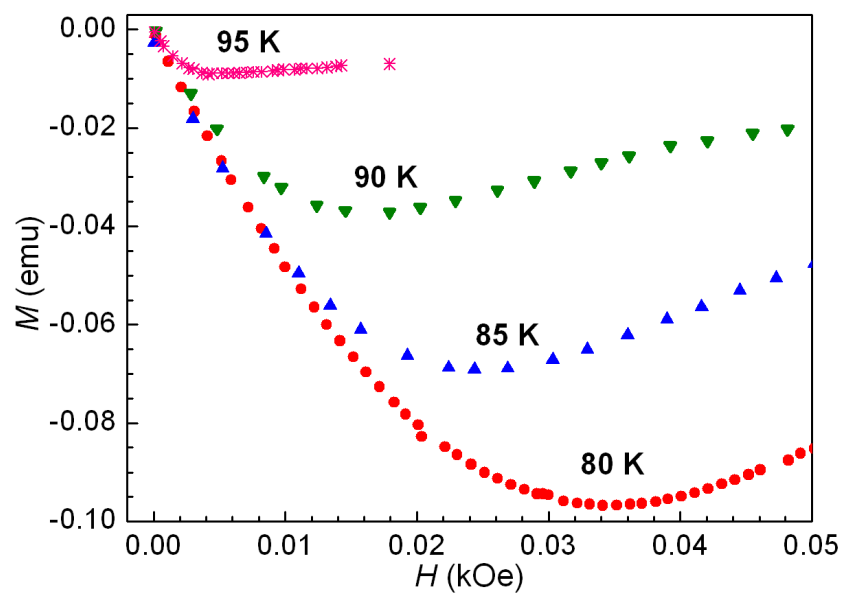

Fig. 2. Virgin curves of the magnetization of $\left(\mathrm{Tl}_{0.5} \mathrm{~Pb}_{0.5}\right) \mathrm{Sr}_{2}\left(\mathrm{Ca}_{0.8} \mathrm{Gd}_{0.2}\right) \mathrm{Cu}_{2} \mathrm{O}_{z} \quad$ superconductor versus the applied magnetic field for several temperatures.

as the percolation models Eq. (4). Both of them fit the experimental data quite well.

The virgin curves of the magnetization as a function of the applied magnetic field for several temperature are shown in Fig. 2. For increasing magnetic field the magnetization reaches a minimum at which the magnetic field penetrates to the center of the superconducting grains. This is the so-called full penetration field $H_{\mathrm{p}}$. These fields increase as temperature decreases. This dependence is shown in Fig. 3. These data were successfully fitted using the linear function because they were obtained within very narrow temperature range close to the critical temperature. In the same figure the magnetic fields in which the maxima of the ac absorption susceptibility were observed are also shown. One can notice that they are well

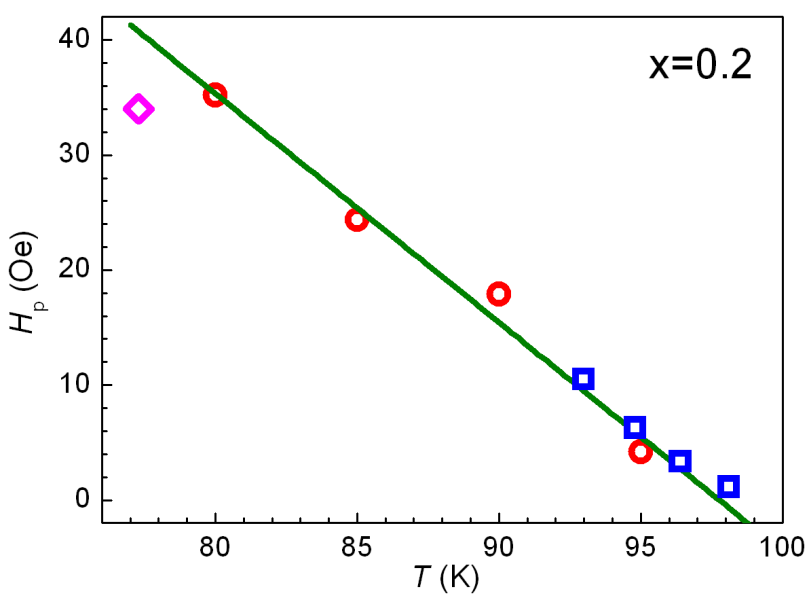

Fig. 3. Penetration fields as a function of temperature of $\left(\mathrm{Tl}_{0.5} \mathrm{~Pb}_{0.5}\right) \mathrm{Sr}_{2}\left(\mathrm{Ca}_{0.8} \mathrm{Gd}_{0.2}\right) \mathrm{Cu}_{2} \mathrm{O}_{z}$. The experimental points came from magnetization (open circles), from ac susceptibility (open squares) [4] and from microwave absorption (open diamond) measurements. Solid line was fitted to the magnetization data.

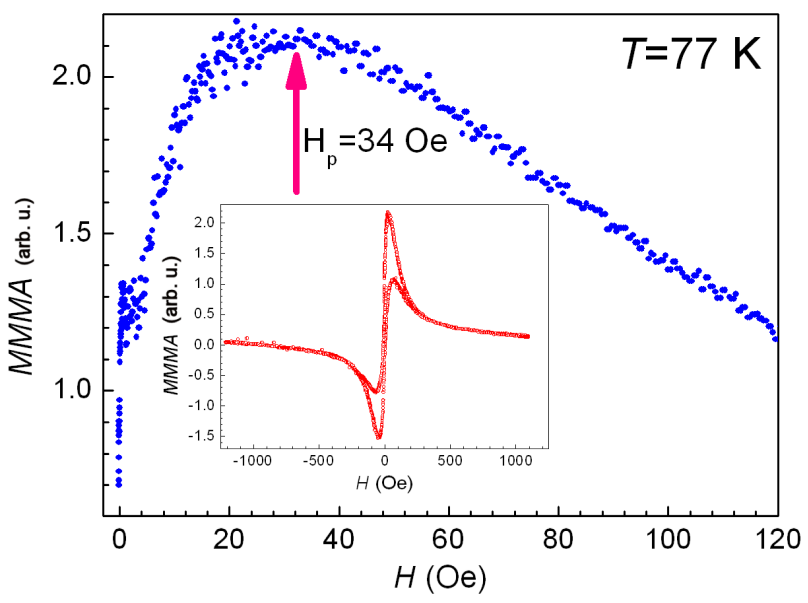

Fig. 4. MMMA as a function of the applied magnetic field of $\left(\mathrm{Tl}_{0.5} \mathrm{~Pb}_{0.5}\right) \mathrm{Sr}_{2}\left(\mathrm{Ca}_{0.8} \mathrm{Gd}_{0.2}\right) \mathrm{Cu}_{2} \mathrm{O}_{z}$ The arrow points out the low field maximum. Inset shows the MMMA loop.

fitted by the same line. That is because these characteristic fields measured by the ac susceptibility as well as by the magnetization describe the same intra-grain regions.

The MMMA as a function of the applied magnetic field at $77 \mathrm{~K}$ is shown in Fig. 4. The MMMA signal is responsible for microwave energy dissipation in inter-granular links. The so-called low field maximum (LFM) appears if the magnetic field entirely penetrates almost all of the inter-granular links. This LFM is $H_{\mathrm{pJ}}=34$ Oe at $77 \mathrm{~K}$ and it fits the full penetration field line obtained from the magnetization as well as from the ac susceptibility measurements (see Fig. 3). 


\section{Summary and conclusion}

The inter- and intra-granular properties of bulk $\left(\mathrm{Tl}_{0.5} \mathrm{~Pb}_{0.5}\right) \mathrm{Sr}_{2}\left(\mathrm{Ca}_{0.8} \mathrm{Gd}_{0.2}\right) \mathrm{Cu}_{2} \mathrm{O}_{z}$ superconductor were measured. The critical current densities obtained from the susceptibility measurements using the Bean model and from the transport measurements are very similar. The critical current versus the applied magnetic field was well fitted using the Kim as well as the percolation models. The penetration fields obtained from the ac susceptibility, the magnetization as well as from the microwave absorption measurements are comparable. These experimental results can be concluded that the superconducting properties of the inter-granular links are similar to the properties of grains in $\left(\mathrm{Tl}_{0.5} \mathrm{~Pb}_{0.5}\right) \mathrm{Sr}_{2}\left(\mathrm{Ca}_{0.8} \mathrm{Gd}_{0.2}\right) \mathrm{Cu}_{2} \mathrm{O}_{z}$ bulk superconductor.

\section{Acknowledgments}

This project was supported by the Faculty of Physics and Applied Computer Science, AGH University of Science and Technology, Cracow, Poland and by the Austrian Science Foundation (Fonds zur Förderung der Wissenschaftlichen Forschung in Österreich, project number 17420-N07).

\section{References}

[1] W.M. Woch, R. Zalecki, A. Kołodziejczyk, O. Heiml, G. Gritzner, Physica C 434, 17 (2006).

[2] W.M. Woch, R. Zalecki, A. Kołodziejczyk, C. Deinhofer, G. Gritzner, J. Alloys Comp. 442, 209 (2007).

[3] C. Deinhofer, G. Gritzner, Supercond. Sci. Technol. 17, 1196 (2004).

[4] W.M. Woch, R. Zalecki, A. Kołodziejczyk, H. Sudra, G. Gritzner, Supercond. Sci. Technol. 21, 085002 (2008).

[5] Y.B. Kim, C.F. Hempstead, A.R. Strand, Phys. Rev. Lett. 9, 306 (1962); Y.B. Kim, C.F. Hempstead, A.R. Strand, Phys. Rev. 129, 528 (1963).

[6] M. Xu, D. Shi, R.F. Fox, Phys. Rev. B 42, 10773 (1990).

[7] W.M. Woch, R. Zalecki, A. Kołodziejczyk, O. Heiml, H. Sudra, G. Gritzner, Acta Phys. Pol. A 114, 99 (2008).

[8] T.B. Doyle, R.A. Doyle, Phys. Rev. B 47, 8111 (1993).

[9] H. Sudra, G. Gritzner, H. Puchinger, V. Kavečanski, Appl. Phys. A 84, 445 (2006).

[10] J. Niewolski, A. Kołodziejczyk, T. Zając, W.M. Woch, Z. Tarnawski, K. Przybylski, T. Brylewski, G. Gritzner, M. Enengl, W. König, O. Heiml, Physica C 388-389, 4 (2003). 